Update article

\title{
Revisiting prophylaxis of homeopathic interventions in Covid 19
}

\section{Aditya Dilipkumar Patil}

Assistant Professor, Department of Homoeopathic Pharmacy, Dr. D. Y. Patil Homoeopathic Medical College and Research Centre, Pimpri, Pune, Maharashtra, India, Pincode - 411018

aditya.patil@dpu.edu.in - https://orcid.org/0000-0003-1537-4403

\begin{abstract}
Severe acute respiratory syndrome coronavirus 2 (SARS-CoV-2) caused by novel beta-coronavirus has emerged as the cause of coronavirus pandemic (COVID-19) declared by the Public Health Emergency of International Concern (PHEIC). Korean oriental medicine, Traditional Chinese Medicine (TCM), and Indian systems of medicine known as AYUSH (Ayurveda, Yoga and Naturopathy, Unani, Siddha and Sowa-Rigpa, and Homeopathy) had implemented various prophylactic measures and interim treatment guidelines in prevention and treatment for COVID -19 cases. Even though there were so many different approaches implemented to break the chain of the epidemic, we have not reached herd effect or herd immunity in the Indian population. In this ongoing COVID-19 pandemic, a specific study on immune markers of IL-6 (Interleukin-6), D-Dimer, Ferritin, CRP (Creactive protein) with SARS CoV-2 specific IgG \& IgM antibodies need to be investigated for generating hard-core evidence for homeoprophylaxis in terms of immunity response. Therefore, there seems to be a need of revisiting the program of homeoprophylaxis in the COVID -19 pandemic. Keywords: Homoeoprophylaxsis, Immunoglobulin, SARS-CoV-2.
\end{abstract}

\section{Background}

Severe acute respiratory syndrome coronavirus 2 (SARS-CoV-2) caused by novel beta-coronavirus has emerged as a cause of coronavirus pandemic (COVID-19) declared by the Public Health Emergency of International Concern (PHEIC) [1]. Polymerase chain reaction (PCR) based investigations are recommended for the diagnosis of positive SARS CoV-2 patients [2]. Korean oriental medicine, Traditional Chinese Medicine (TCM), and Indian systems of medicine known as AYUSH (Ayurveda, Yoga and Naturopathy, Unani, Siddha and Sowa-Rigpa, and Homeopathy) had implemented various prophylactic measures and interim treatment guidelines in prevention and treatment for COVID -19 cases [3]. Prophylactic measures (including community measures \& lockdown) like a Face mask, steam inhalation, mouth wash, hand sanitization, social distancing, UV radiation; Quarantine \& Isolation, and supplementary products for the immune booster (Vitamin C, Zinc, and Vitamin D), herbal products (Curcumin longa extract, Ocimum sanctum extract, Eucalyptus drops, etc) were used rampantly [4].

\section{Prophylactic interventions of TCM/AYUSH \& ICMR -}

Modern medicine implemented the use of Anti-viral (Faviparavir \& Remdesivir), Anti-parasitic (Ivermectin), Antibiotic (Azithromycin, Doxycycline), Steroids (Dexamethasone \& Methylprednisolone), Anti-pyretic (Paracetamol) and followed with Vaccines [mRNA, Viral like Particle (Spike Glycoprotein), Inactivated, Recombinant or Conjugated], Convalescent plasma therapy for flattening the curve of COVID-19 cases [5]. Homeoprophylaxis imposed by an advisory of the Ministry of AYUSH named Arsenic album 30C was also intervened in the COVID-19 pandemic. (6) Other homeopathic medicines like Camphor, Zinc muriaticum, Antimonium tartum, Bryonia alba, 
Cinchona officinalis, Phosphorus, Gelsemium sempervivum, Mercurius solubilis were also intervened with expert opinions from the homeopathic fraternity [7-9] These guidelines were formed from sources of Case Reports, Materia Medica Pura, Reportorial analysis of COVID-19 cases (Individualized method and Genus Epidemicus), however documenting as per the guidelines of World Health Organisation (WHO) and Indian Council of Medical Research (ICMR) for an evidence based medicine still persist a challenge [10]. With such different approaches imposed in cases of Asymptomatic, Mild, Moderate \& Severe COVID -19 cases, there still a lacks herd effect or herd immunity in the Indian population. In such a catastrophic situation new variants of coronavirus [E484K (India), B.1.1.7 (United Kingdom), B.1.351 (South Africa), P.1 (Brazil), L452R (California)] are more concerned [11].

\section{Immunology and Prophylaxis}

Prophylactic measures are defined as, wherever possible, preventing the infection caused by virus or bacteria, etc, i.e., preventing entry of causative organism in the host cells, through sterilizing immunity protection mediated by antibodies. The adaptive response has both cellular and humoral responses, wherein T cells for cellular adaptive immune response \& antibodies for humoral response are mediators [12]. The above-mentioned prophylactic measures however with their previous literature based on in-vitro, in-vivo, and molecular docking studies, experimented with various models that were collaborated and implemented in achieving both cellular and humoral immunity in COVID-19 cases. Unfortunately, the second wave of COVID-19 has again increased the curve of positive cases in such a drastic way if compared with the annual doubling time; the rate of recovery has reduced with the rate of confirmed positive cases, with reduced mortality but increase in infection spread in Indian population [13].

\section{Future perspective}

The second wave of COVID-19 cases explains the struggle of the above-mentioned prophylactic measures in terms of detecting seroconversion of both SARS-CoV-2 IgG (Immunoglobulin G) \& IgM (Immunoglobulin M) in newly suspected and previously infected patients of SARS-CoV-2. Serological tests for viral antibodies provide an important tool to diagnose previous exposure to the virus [14]. Various studies are ongoing on measuring the sensitivity and specificity of seropositive testing in SARS CoV-2 patients, wherein some have got concluded and some are still under the process of data verification [14]. These seropositive surveys might provide an additional contribution in measuring the longitudinal effects of prophylaxis in terms of the duration of lasting SARS-CoV 2 specific IgG \& IgM antibodies.

\section{Homoeoprophylaxsis: SARS-CoV 2 specific IgG \& IgM antibodies perspective}

Homeoprophylaxis on the other hand needs rigorous data specific to SARS-CoV 2 specific IgG \& IgM antibodies induced after the ingestion of homeopathic medicines. Undoubtedly homeopathic medicines had shown prophylactic effects in various epidemic outbreaks like Leptospirosis, Dengue, Japanese encephalitis, etc. but there seems to be deficient evidence in terms of viral or bacterial specific immune response measurement [15-17]. In the ongoing COVID-19 pandemic, a specific study on immune markers of IL-6 (Interleukin-6), D-Dimer, Ferritin, CRP (C-reactive protein) with SARS CoV-2 specific IgG \& IgM antibodies might bring hard-core evidence for homeoprophylaxis in terms of immunity response. Further data can be compared with vaccine efficacy or any medicated treatment mode and might be useful in implementing at the appropriate duration of time if studied meticulously on incubation periods correlating with different time intervals. However, the chances of false positive cases must also be concerned in designing such studies as it might affect the results 
of prophylaxis. Public health professionals with infectious disease experts understand the transmission dynamics of SARS CoV-2 infected patients for preventive and treatment modalities. In such circumstances, any misleading claim from TCM, or AYUSH might lead to detrimental effects encountering this epidemic. CCRH (Central Council for Research in Homoeopathy) experts with the homeopathic academic institute are conducting research in the domain of randomized controlled clinical trials, developing corona virus nosodes, and designing a case repository database for COVID19 cases. Collaborating with public health and infectious diseases experts to develop potential leads, and research protocols for the scientific peer-review process (AYUSH Task Force Committee for COVID-19) might provide link of strength and limitation of the system in building up evidence in Serological surveillance of IgG and IgM immunoglobulin's specific to SARS-CoV-2 infected individuals.

\section{Source of funding}

None

\section{Conflict of Interest}

None

\section{References}

[1] Centers for Disease Control and Prevention 2019 Novel Coronavirus (2019-nCoV), (2020), https://www.cdc.gov/coronavirus/2019-ncov/cases-in-us.html, Accessed 9th Mar 2020.

[2] Buddhisha Udugama et.al. Diagnosing COVID-19: The Disease and Tools for Detection, ACS Nano 2020, 14(4), 3822-3835, DOI: 10.1021/acsnano.0c02624.

[3] M.M. Zhang, X.M. Liu, L. He, Effect of integrated traditional Chinese and Western medicine on SARS: a review of clinical evidence, World J Gastroenterol, 10 (2004), pp. 3500-3505, 10.3748/wjg.v10.i23.3500.

[4] Z. Qi, WHO traditional medicine strategy 2014-2023, World Health Organization, Geneva (2013).

[5] Juul S, Nielsen EE, Feinberg J, Siddiqui F, Jorgensen CK, Barot E, et al. (2021), Interventions for the treatment of COVID-19: Second edition of a living systematic review with meta-analyses and trial sequential analyses (The LIVING Project). PloS ONE 16(3): e0248132. https://doi.org/10.1371/journal.pone.0248132.

[6] Advisory for Corona virus. Homoeopathy for prevention of Corona virus infections Unani medicines useful in symptomatic management of Corona virus infection. Pib.gov.in; 2020. Posted On: 29 JAN 2020 by PIB Delhi. https://pib.gov.in/PressReleasePage.aspx?PRID=1600895. [Accessed 08 March 2020]

[7] Chaudhary A, Khurana A. A review on the role of Homoeopathy in epidemics with some reflections on COVID-19 (SARS-CoV-2). Indian J Res Homoeopathy 2020;14:1009

[8] Fujino FMSDC et.al. Antimonium Tartaricum as a Possible Homeopathic Prophylactic Remedy in the COVID-19 Epidemic. Homeopathy. 2021 Apr 14. Doi: 10.1055/s-0041-1725060. 
[9] Azis SP, Kaur H. Enabling use of Homoeopathy in India's preparedness for pandemic/epidemic situation like COVID-19. Indian J Res Homoeopathy 2020; 14: 14351.

[10]Bipin Jethani et.al. Clinical Characteristics and Remedy Profiles of Patients with COVID-19: A Retrospective Cohort Study, The Faculty of Homeopathy, 2021, DOI https://doi.org/10.1055/s-0040-1718584.

[11]World Health Organization, COVID-19 new variants: Knowledge gaps and research. https://www.who.int/publications/m/item/covid-19-new-variants-knowledge-gapsand-research. [Accessed 25th March 2021].

[12]Silverstein AM. Cellular versus humoral immunity: determinants and consequences of an epic nineteenth century battleIn: Silverstein AM, editors. A History of Immunology. 1st ed New York; Academic Press Inc; 198938-58.

[13]Alok Tiwari, Modelling and analysis of COVID-19 epidemic in India, Journal of Safety and Resilience 1 (2020) 135-140, https://doi.org/10.1016/j.jnlssr.2020.11.005.

[14] Nakano, Y., Kurano, M., Morita, Y. et al. Time course of the sensitivity and specificity of anti-SARS-CoV-2 IgM and IgG antibodies for symptomatic COVID-19 in Japan. Sci Rep 11, 2776 (2021). https://doi/10.1038/s41598-021-82428-5.

[15]Isaac Golden, The place of homeoprophylaxis in modern medicine, Homeopathic Links; Guest Editorial. https://dx.doi.org?10.1055?s-0036-1583537.

[16]Delgado JP et.al. Large-scale homoeoprophylaxis as an add-on measure to prevent COVID-19 disease: Cuban preliminary experiences. Indian J Res Homoeopathy 2020; 14:293-4

[17]Oberai P et.al. Effectiveness of Homeopathic Medicines as Add-on to Institutional Management Protocol for Acute Encephalitis Syndrome in Children: An Open-Label Randomized Placebo-Controlled Trial. Homeopathy. 2018 Aug:107(3):161-171. doi:10.1055/s-0038-1656715.

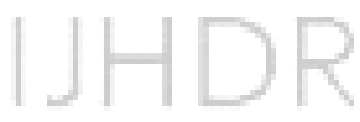

(C) International Journal of High Dilution Research.

Not for commercial purposes. 